\title{
The Understanding of Pitch Among Hearing and Deaf Adults
}

\author{
Boyan Ruan ${ }^{*}$, Ziyan Yang ${ }^{2+}$ and Zilu $\mathrm{Jin}^{3+}$ \\ ${ }^{1}$ Beijing International Bilingual Academy Guiyang Campus, Guiyang, Guizhou, 550000, China \\ ${ }^{2}$ Baoji Middle School, Baoji, Shaanxi, 721013, China \\ ${ }^{3}$ Fontbonne Academy, Milton, MA, 02186, America \\ *Corresponding author. Email: Boyan_Ruan@outlook.com
}

${ }^{+}$These authors contributed equally to this work and should be considered co-second authors.

\begin{abstract}
In this work, it is suggested that even the deaf could understand pitch through linguistic interaction, feelings of vibration, observation of facial expressions; or a combination of them all. This proposed study will test these hypotheses by using sound-reproduction tasks and provide evidence for the practical implication of how the understanding of pitch may help the deaf in everyday life.
\end{abstract}

Keywords: pitch, the deaf, the hearing, vibration, facial expression, linguistic interaction

\section{INTRODUCTION}

It is widely believed that experience is one of the most important ways of learning. Because of this, traditional empiricists like Locke thought that concepts like colour and pitch were inaccessible for the blind or deaf (Locke \& Pringle-Pattison, 1924) [1]. Particularly, modern cognitive theories claim that first-person experience is the central way of understanding sensory phenomena. Hence, without the help of first-person experience, understanding pitch might be extremely difficult or even impossible for deaf people (Prinz, 2005) [2].

Though these arguments make sense intuitively, we should also notice that except for first-person experience, there are still other approaches to learning basic concepts. For example, people can learn the concept of colour from linguistic interactions (Kim et al., 2021) [3], and learn rhyme from observing the movements of others' lips when they are speaking, or by using orthographical methods (Hanson, \& McGarr, 1989) [4].

Like how the blind learnt to understand colour, deaf people may be able to learn the concept of pitch from communications with the hearing ones. They can directly learn the knowledge of pitch by the explanations of it and its relevant facts. For example, Moreover, studies have shown that language experiences, such as the acquisition of tone languages (languages that use variations in the pitch of monosyllabic words to represent different meanings, e.g., Mandarin), would sharpen the response of neurons tuned for processing pitch contours (Krishnan, et al., 2005) [5], and enhance pitch representation at the brainstem level (Xu et al., 2006) [6]. When they are speaking languages, those with conduction deafness could hear themselves and feel the change in tones or pitch, which can facilitate them to understand pitch as well.

Alternatively, the famous case of Ludwig van Beethoven, one of the greatest German musicians of the $18^{\text {th }}$ century who accidentally became deaf and used a solid stick to conduct himself to hear different notes, implies that the deaf could possibly learn to identify different levels of pitch from feeling the vibration of different objects when they are making sounds. In contrast, the largest difference between the situation of Beethoven and congenitally deaf people is that Beethoven had already known what pitch was before he got deaf, while those with congenital hearing loss did not. In this case, to learn the concept of pitch in this way, proper guidance by hearing people may be needed since we are not sure how possible the deaf may know that they can hear sounds in this way.

Additionally, the observation about the expression of people when they are speaking or singing may enable the deaf to enhance the understanding of pitch as well. Studies have shown that people usually use different pitches to express different types of emotions (Scherer, 
1974) [7]. For instance, high pitch represents activity, potency, anger, etc., while low pitch signals pleasantness, boredom, or sadness.

Simultaneously, people always apply facial expressions involuntarily to show their emotions. Although deaf people lack access to conventional cues, they would unconsciously use facial expressions to represent basic emotions in a similar way to the hearing ones' (Eibl-Eibesfeldt, 1972) [8]. Thus, it is not difficult for the deaf to correlate 'happiness' with a smile and high pitch, or correlate 'sadness' with corners of the mouth down and low pitch. But this suggestion is set in a premise that they are able to hear themselves and have already learnt how to speak. In this case, once they noticed the cooccurrence of the unconscious reaction of changing their tones when speaking and showing correspondent expressions when feeling specific emotions, they would easily know that they are associated and apply this finding to interpret the change in emotions of others, as well as applying it to facilitate communication with others.

In a holistic view, it could be argued that the deaf may not just understand the concept of pitch with the help of one single factor, but a combination of various methods. They may first learn the existence of a concept of pitch by linguistic interaction with hearing people; then feel how high and low pitch differs from each other by feeling the different frequency of vibrations of sounding objects; and finally learn to correlate emotion with corresponding facial expression and levels of pitch, which is a stage of using the concept in practice in order to help them better engaged into daily communications with others - they can vary their tones and pitches of speech to better express their own feelings. This proposed study will test the hypotheses above by conducting an experiment using independent groups design and provide the first evidence about if and how deaf adults understand pitch in a similar way to that of the hearing ones.

\section{SPECIFIC AIMS}

1. To test whether the deaf have the understanding of pitch;

2. To investigate what specific factors facilitated them to understand pitch, if they do understand that.

\section{IMPLICATIONS}

In fact, many deaf people cannot hear what others say, nor can they hear themselves, so they become deaf-mutes. However, there are still some people who can speak, but what they say is not well understood by others. There might be problems in pronunciation and intonation, etc. If the hypothesis that deaf people can also understand pitch is true -- deaf people can understand the concept of pitch through verbal communication, facial expressions, feeling frequency, etc., then deaf people who can speak can be trained in pitch control, to some extent to express emotions more accurately to reduce their communication difficulties. For deaf-mutes, the community can also provide them with tactile and visual training to better communicate with others without misunderstanding due to differences in pitch recognition.

Many studies have shown that deaf students have difficulties in music lessons, including the inability to sing the correct pitches or notes (Meeks, 2020) [9]. If they can understand pitch, it may be possible to teach them music appreciation and composition (because some of them may be able to imagine sounds), and even to teach them to sing, helping them solve their dilemmas in music lessons and making music classes accessible to more deaf people.

Deaf people also have a certain understanding of the phonological structure of rhyme (Hanson,1989) [4], so combined with the understanding of pitch, this will help them to achieve in fields like poetry.

\section{PROPOSED STUDY}

The proposed study addresses:

(i.) the effect of vibrations of objects on pitch reproduction ability;

(ii.) the effect of facial expression of participants on pitch understanding ability;

(iii.) the effect of linguistic interaction of participants on pitch understanding ability.

First, the previous study revealed the possible basis on which deaf individuals could generate rhymes is speech information--either the visual characteristics of words or the lips of speakers (Dodd \& Hermelin, 1977) [10]. The present study will test whether deaf adults have a shared understanding of pitch with hearing ones. Second, we will determine whether deaf people can better reproduce pitch by feeling vibrations, observing facial expressions while engaging in linguistic interactions.

To address these questions, we will ask Group 1 to feel the vibrations of an audio player when playing sounds with different levels of pitch and reproduce the similar vibrations of vocal cords. Group 2 will observe facial expressions of hearing people (an opera singer) when producing pitches, and then try to reproduce the voices. This will allow us to probe the specific nature of deaf adults' knowledge of the mechanisms that give rise to object pitch.

In contrast, there will be a control group that consists of deaf people who receive no training and receive the same task as the experimental groups. 
After these procedures above, we will ask all participants to reproduce the pitch with any methods that would help in order to probe the influences of vibrations, linguistic interactions and facial expression. Therefore, we will compare to what extent participants in different groups can reproduce sounds that are matched to the original ones in their levels of pitch to show whether deaf people can benefit 1 from these trainings.

\section{METHOD}

\subsection{Participants}

We will recruit 30 congenitally deaf participants. The sampling technique will be volunteer sampling in which we will post an advertisement in a magazine mainly for deaf people.

All of them have within the normal range of intelligence (between 80 and 115). This will be tested by using Wechsler Intelligence Scale before the experiment starts. Their ages should range between 16 and 40 years old. Also, all deaf participants should report no experience with pitch, academic vocal training. The selection of subjects was limited to people with sensori-neural hearing losses displaying a typical audiometric pattern of increased hearing loss with higher frequencies, with a loss in the better ear (pure-tone average) no greater than $105 \mathrm{~dB}$ (Ford, T. A., 1988) [11].

All participants will be randomly assigned into the three groups (Group 1, Group 2, and the control group), using a random number generator. Each group will contain 10 participants.

\subsection{Training sessions}

After receiving an IQ test by answering Wechsler Intelligence Scale, each participant in Group 1 will be asked by a hearing trainer to feel the vibrations of the audio player when playing songs of the same opera singer when expressing different moods. They will only hear the sounds without looking at the visual contents. The materials will be consistent with the ones involved in the training for Group 2. This section will last for 15 minutes for every participant. After that, they will be asked to reproduce the sounds by using one hand to feel the vibration of the audio player and the other hand to feel the vibration of their vocal cords.

Group 2 will receive training given by the same hearing trainer to observe the hearing people's facial expressions in process of producing the three levels of pitch without linguistic interaction. They will watch a video about the same opera singer singing with different levels of pitch and using different expressions to show correspondent emotions. The emotions involved will be the 6 basic emotions identified by Ekman (1992): fear, anger, sadness, happiness, disgust, and surprise; since they are already found to be universal facial expressions of emotions that are shared among humans and even with primates [12]. This will be chosen in order to prevent possible extraneous variables such as people's 'Theory of Mind', the ability to recognize that others have different feelings, beliefs, knowledge and desires than themselves; or empathy, the ability to understand the emotions of others and feel in the same ways. In the next step, they will be asked to reproduce the voices made by the opera singer while looking at their own faces through a mirror provided and trying to imitate the facial expressions of the singer.
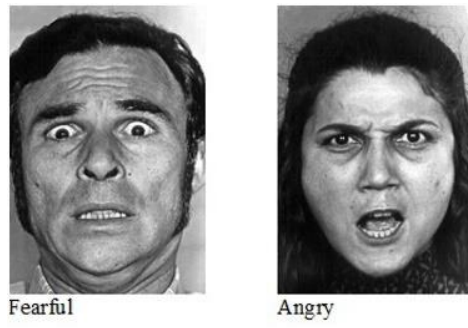

Angry
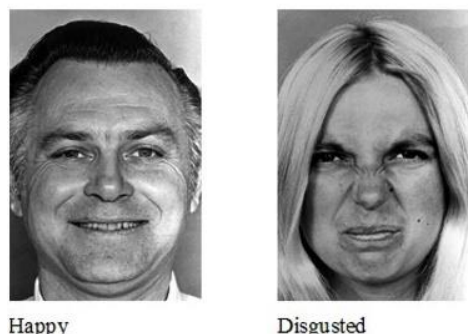

Disgusted
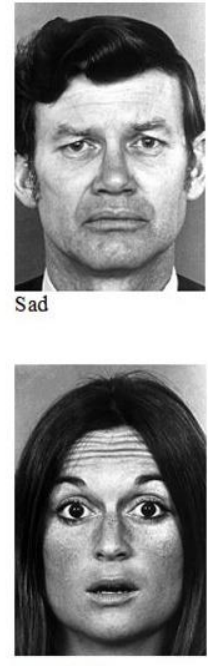

Surprised
Fig.1 Basic Emotions identified by Ekman (1992)

\subsection{Testing session}

Then in the testing session, we will ask all groups of participants to reproduce the pitches made by an audio player, a piano, and a confederate. Each will consist of 6 trials including 2 sounds with 'low' pitch, 2 sounds with 'medium' pitch, and 2 sounds with 'high' pitch.

During the process, they will be allowed to use any methods that would help them to accurately reproduce the sound, such as using a hand to feel the vibration of the audio player. These will be operationalised by conducting a semi-structured interview after the testing session. The interview will contain fixed questions, e.g., 'what did you do in the test to help reproduce pitch accurately?'; and some follow-up questions according to the responses of the participants to the former ones. The results will be analysed by content analysis. Its validity will be checked by recording the testing stage and use as a reference.

The original sounds and responses will be recorded in audio and the compatibility between their levels of pitch will be judged by a group of 5 judges. A response is only considered to be 'matched' when at least 4 judges agree that it is matched with the original sound in the levels of pitch; otherwise, it is regarded as 'unmatched'. The score of each participant on the reproduction of sound task is 
calculated by subtracting the number of unmatched answers from the number of matched answers.

\subsection{Predictions}

According to educators of the deaf train deaf child's ability to enjoy music through the tactile sense (Ford, T. A., 1988) [11]. Thus, see Fig.2, We predict that in the reproduction task from an audio player, the performance of Group 1 and Group 2 will be significantly better than the control group, as the trainings are supposed to work.

In the reproductions task from the sounds made by a piano, we predict that Group 1 will make inferences of pitches according to feeling instruments' vibrations, deaf people can make similar pitch levels using their vocal cords. Therefore, Group 1's mean score should be higher than the others, followed by that of Group 2 and the control group.

An analysis of the facial features shows a strong correlation between eyebrow position and sung pitch - consistent with the role of eyebrows in signaling aggression and appeasement (Huron et al., 2009) [13]. We predict that Group 2's pitch ratings would be correlate with the extent of facial expression exaggerations. For the task to reproduce the sounds made by a confederate, Group 2's mean score should be higher than those of Group 1 and the control group.

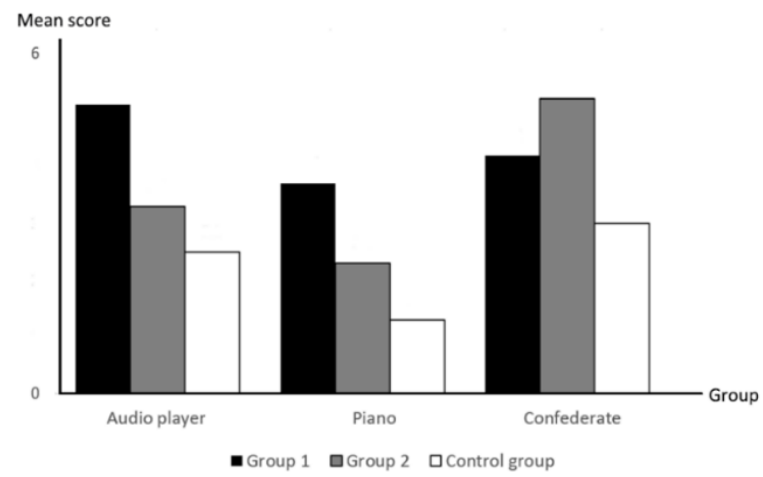

Fig. 2 Predictions about the Mean Scores of Each Group

\section{CONCLUSION}

If it turns out that deaf people can perceive pitch through communication with others, through thinking, through direct sensory experience, then pitch control training for deaf people should be initiated. This is also a deaf ability that should not be wasted. Communities can communicate (language, writing...) to deaf people with laryngeal vocal cord use to control pitch: teach them to try to relax their laryngeal muscles and try to make sounds, and if they do, tell them it's low pitch; Then try to tighten their muscles and indicate that this is a high pitch. Deaf people cannot know what sounds they are making because of their hearing impairment, but they can develop muscle memory by practicing muscle contractions in the throat. By associating muscle memory with pitch and emotion, they were able to overcome the pitch barriers in their communication. For deaf-mutes, who also have the ability to understand pitch, what the community can promote is training in their understanding of facial expressions. Or, if possible, a portable frequency display that can be used to judge when they are sometimes unsure of others' emotions, thus increasing communication efficiency. In addition, what else advantages can be brought by the understanding of pitch to the deaf in daily life?

If it turns out that deaf people can't understand pitch, this will further prove the importance of first-person experience for cognition. This further suggests that direct auditory contact is much more effective than verbal or visual contact in conveying pitch concepts. However, why first-person experience is critical in the understanding of pitch, but is less important in the understanding of color?

Hearing people can understand pitch, and we are going to see if deaf people can understand pitch through experiments. So, what particular neural mechanisms are underlying pitch understanding? And do these mechanisms differ between hearing and deaf people?

\section{REFERENCES}

[1] Locke, J., \& Pringle-Pattison, A. S. (1924). An Essay Concerning Human Understanding... Abridged and Edited by AS Pringle Pattison. Oxford.

[2] Prinz, J. J. (2005). The return of concept empiricism. In Handbook of categorization in cognitive science (pp. 679-695). Elsevier Science Ltd.

[3] Kim, J. S., Aheimer, B., Manrara, V. M., \& Bedny, M. (2021). Shared understanding of color among sighted and blind adults. Proceedings of the National Academy of Sciences, 118(33).

[4] Hanson, V. L., \& McGarr, N. S. (1989). Rhyme generation by deaf adults. Journal of Speech, Language, and Hearing Research, 32(1), 2-11.

[5] Krishnan, A., Xu, Y., Gandour, J., \& Cariani, P. (2005). Encoding of pitch in the human brainstem is sensitive to language experience. Cognitive Brain Research, 25(1), 161-168.

[6] Xu, Y., Krishnan, A., \& Gandour, J. T. (2006). Specificity of experience-dependent pitch representation in the brainstem. Neuroreport, 17(15), 1601-1605.

[7] Scherer, K. R. (1974). Acoustic concomitants of emotional dimensions: Judging affect from synthesised tone sequences. In S. Weitz (Ed.), 
Nonverbal communication (pp. 249-253). New York: Oxford University Press.

[8] Eibl-Eibesfeldt, I. (1972). 11. SIMILARITIES AND DIFFERENCES BETWEEN CULTURES IN EXPRESSIVE MOVEMENTS. Non-verbal communication, 297.

[9] Meeks, Robyn S. (2020) "Inside the Music Classroom: Deaf Students," The Corinthian: Vol. 20, Article 5.

[10] Dodd, B., \& Hermelin, B. (1977). Phonological coding by the prelinguistically deaf. Perception \& Psychophysics, 21(5), 413-417.

[11] Ford, T. A. (1988). The effect of musical experiences and age on the ability of deaf children to discriminate pitch. Journal of Music Therapy, 25(1), 2-16.

[12] Ekman, P. (1992). An argument for basic emotions. Cognition \& emotion, 6(3-4), 169-200.

[13] Huron, D., Dahl, S., \& Johnson, R. (2009). Facial expression and vocal pitch height: Evidence of an intermodal association. Empirical Musicology Review, 4(3), 93-100. 